\title{
Consensus development on the essential competencies for Iranian public health nutritionists
}

\author{
Farzaneh Sadeghi-Ghotbabadi ${ }^{1}$, Elham Shakibazadeh ${ }^{2}$, Nasrin Omidvar ${ }^{2, *}$, \\ Fathieh Mortazavi ${ }^{3}$ and Fariba Kolahdooz ${ }^{4}$ \\ ${ }^{1}$ Nutrition Department, Ministry of Health and Medical Education, Tehran, Islamic Republic of Iran: ${ }^{2}$ Department of \\ Community Nutrition, National Nutrition and Food Technology Research Institute; and Faculty of Nutrition Sciences \\ and Food Technology, Shahid Beheshti University of Medical Sciences, 46 West Hafezi Street, Farahzadi Boulevard, \\ Shahrak Qods, 1981619573 Tehran, Islamic Republic of Iran: ${ }^{3}$ School of Medical Education, Shahid Beheshti \\ University of Medical Sciences, Tehran, Islamic Republic of Iran: ${ }^{4}$ Aboriginal and Global Health Research, \\ Department of Medicine, University of Alberta, Edmonton, Alberta, Canada
}

Submitted 19 July 2013: Final revision received 4 March 2014: Accepted 3 April 2014: First published online 27 May 2014

\begin{abstract}
Objective: To assess key experts' opinion regarding essential competencies required for effective public health nutrition practice within the health-care system of Iran. Design: Qualitative study using the modified Delphi technique through an emaildelivered questionnaire.

Setting: Iran.

Subjects: Fifty-five experts were contacted through email. The inclusion criterion for the study panel was being in a relevant senior-level position in nutrition science or public health nutrition in Iran.

Results: In the first round, forty-two out of fifty-five experts responded to the questionnaire (response rate $=76 \%$ ). A sixty-five-item questionnaire was designed with nine competency areas, including 'nutrition science', 'planning and implementing nutritional interventions', 'health and nutrition services', 'advocacy and communication', 'assessment and analysis', 'evaluation', 'cultural, social and political aspects', 'using technology' and 'leadership and management'. All experts who had participated in the first round completed a modified version of the questionnaire with seventy-seven items in the second round. The experts scored 'nutrition science' as the most essential competency area, while more applied areas such as 'management and leadership' were less emphasized. In both rounds, the mean difference between the opinions of the necessity of each area was $5.6 \%$. Conclusions: The Iranian experts had general agreement on most of the core competency areas of public health nutritionists. The results indicated the need for capacity building and revisions to educational curricula for public health nutritionist programmes, with more emphasis on skill-based competency development.
\end{abstract}

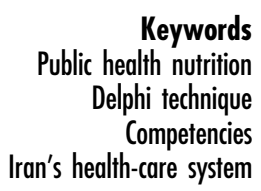

Based on a broad definition, public health nutrition (PHN) refers to the practice of preventing nutrition-related diseases and conditions, as well as promotion and support to improve the nutritional health of a population. Hughes (2003) has defined PHN as the art and science of promoting population health status via sustainable improvements in the food and nutrition system. It is a set of comprehensive and collaborative activities, ecological in perspective and interdisciplinary in scope, including environmental, educational, economic, technical and legislative measures $^{,(1)}$. Therefore, public health nutritionists are expected to apply scientific principles and methods in the field of nutrition to influence factors affecting food supply and dietary habits, enhance nutritional status and prevent chronic diseases, and show leadership in nutrition and nutrition-related issues. They are also expected to design, coordinate, implement and evaluate a range of population interventions to improve the well-being of individuals, communities and populations as a whole through better dietary practices ${ }^{(1,2)}$.

The Iranian people are experiencing a nutritional and lifestyle transition. The coexistence of over- and undernutrition is a common phenomenon of growing public health concern while, simultaneously, an increasing prevalence of diet-related chronic diseases is occurring due to urbanization and lifestyle changes ${ }^{(3,4)}$. Utilizing the 
health-care system and nutritionists working within the system would therefore be an effective strategy to reduce nutritional problems. Currently, there are 373 nutritionists in the primary health-care system in Iran working as public health nutritionists; however, their roles are mainly in supervision. The present study is the first one conducted in the nation to assess the opinions of key experts regarding essential competencies required for effective PHN practice within the Iranian health-care system. The results can serve as the basis for both planning job training programmes and appropriate revisions to present training curricula in the future.

\section{Methods}

The current qualitative study took place from February to October 2012. The experts were purposely chosen for the study based on their expertise and reputation. There is little evidence on the effect of selecting a larger panel on the reliability or validity of consensus processes ${ }^{(1)}$.

An email contact list was compiled based upon the inclusion criterion of the study. The participants were invited to take part in the study via email. The list was completed using snowball sampling. The inclusion criterion for choosing the study's panel was being in a relevant senior-level position in nutrition science or PHN in Iran. Overall, fifty-five experts were recruited for the study, including eleven managers and four health officers working in the Ministry of Health, thirty-one faculty members in nutrition departments of public universities of medical sciences and nine public health nutritionists working at the managerial level of the health-care system.

The Delphi technique involves a series of three survey rounds among a panel of experts to reach a consensus. The consensus is considered to be reached if group ratings on suggested competencies vary by $\leq 10 \%$ between the rounds ${ }^{(5)}$. In our study, the mean difference, in terms of opinions expressed pertaining to the necessity of each area, after two rounds was $5.6 \%$; therefore, the Delphi process was stopped after two rounds.

The Delphi technique is a process of iteration and managed feedbacks ${ }^{(5)}$. In the first round, the goal was to ask general questions to gain a broad understanding of the experts' viewpoints. We structured a sixty-five-item questionnaire consisting of nine competency areas based on the literature ${ }^{(1,5-9)}$, as well as a job description for Iranian public health nutritionists. The competencies included nutrition science (fourteen items), planning and implementation of nutritional interventions (four items), health and nutrition services (six items), advocacy and community (six items), assessment and analysis (twelve items), evaluation (five items), cultural, social and political aspects (six items), using technology (three items), and leadership and management (nine items). The responses to the questions were ranged according to a three-point
Likert scale as 'essential', 'not necessary but useful' and 'unnecessary'.

A panel of eight key experts evaluated the validity of the content of the questionnaire. Members of the panel included the faculty members of four nutrition departments, a faculty member in health education, a general practitioner, an MSc in Nutrition from the Ministry of Health and a public health nutritionist working at the managerial level of the health-care system. Internal consistency of the questionnaire was assessed using Cronbach's alpha coefficient.

The Delphi technique requires close collaboration with the participants. The objectives and importance of the study, along with the Delphi technique, were thoroughly explained in letters sent to the participants; however, most of the participants in our study were experts relatively familiar with the Delphi technique. Additionally, we explained the methods of answering the questionnaire to the respondents via telephone or in person (face to face). After sending the first-round questionnaire, we collected the written comments from participants with regard to the proposed questions. Based on those comments, the second round of questions delved deeper into the topic, developing twelve new questions (a total of seventy-seven items) based on the previously stated comments. The participants were sent the results from the first round along with the second-round questionnaire. In both rounds, the participants returned the completed questionnaires as an email attachment, fax or a hard copy.

The ethics committee of Shahid Beheshti University of Medical Sciences approved the study's protocol. The participants completed and signed the consent forms for the study and were ensured that their responses would be confidential.

The results were collated and summarized. Focusing on the areas that were agreed upon by the experts, common ground was established and a consensus was met, hence removing any irrelevant content. No standard for achieving consensus is available in the literature ${ }^{(5)}$; therefore, the arbitrary consensus standard was set at $\geq 80 \%$ agreement (i.e. majority rule) in each Delphi round. This approach for defining stability of the consensus has been used in previous studies $^{(5,6)}$. The statistical software package PASW Statistics version 18 was used to analyse the data and descriptive statistics were used to compare response distributions.

\section{Results}

In the first round, forty-two out of fifty-five experts responded to the questionnaire (response rate $=76 \%$ ). Thirteen experts did not participate in the study, including eleven faculty members and two managers whose main reasons for not taking part were not having time to collaborate and respond to the emails. All participants ( $n$ 42) who had completed the first round, responded and 
Table 1 Characteristics of the panellists who took part in both rounds of the Delphi study on essential competencies of public health nutritionists, by position, education and gender; Iran, February-October 2012

\begin{tabular}{|c|c|c|c|c|c|c|c|c|c|c|}
\hline & \multicolumn{8}{|c|}{ Position } & & \\
\hline & \multicolumn{2}{|c|}{ Manager } & \multicolumn{2}{|c|}{ Faculty member } & \multicolumn{2}{|c|}{ Health officer } & \multicolumn{2}{|c|}{ Public health nutritionist } & \multicolumn{2}{|c|}{ Total (row) } \\
\hline & $n$ & $\%$ & $n$ & $\%$ & $n$ & $\%$ & $n$ & $\%$ & $n$ & $\%$ \\
\hline \multicolumn{11}{|l|}{ Education } \\
\hline BSc in Nutrition & 0 & 0 & 0 & 0 & 1 & 17 & 5 & 83 & 6 & 14 \\
\hline MSc in Nutrition & 0 & 0 & 3 & 43 & 1 & 14 & 3 & 43 & 7 & 17 \\
\hline $\mathrm{PhD}$ in Nutrition & 3 & 16 & 14 & 74 & 1 & 5 & 1 & 5 & 19 & 45 \\
\hline General practitioner & 1 & 50 & 0 & 0 & 1 & 50 & 0 & 0 & 2 & 5 \\
\hline Medical specialist & 3 & 75 & 1 & 25 & 0 & 0 & 0 & 0 & 4 & 10 \\
\hline Other PhD & 2 & 50 & 2 & 50 & 0 & 0 & 0 & 0 & 4 & 10 \\
\hline \multicolumn{11}{|l|}{ Gender } \\
\hline Male & 3 & 20 & 11 & 73 & 0 & 0 & 1 & 7 & 15 & 36 \\
\hline Female & 6 & 22 & 9 & 33 & 4 & 15 & 8 & 30 & 27 & 64 \\
\hline Total (column) & 9 & 21 & 20 & 48 & 4 & 10 & 9 & 21 & 42 & 100 \\
\hline
\end{tabular}

completed the second-round questionnaire (response rate $=100 \%$ ). Table 1 shows the general characteristics of the participants in the study.

Internal consistency of the questionnaire for all the competency areas was satisfactory. The highest reliability score (Cronbach's $\alpha$ ) was observed on 'leadership and management' (first round's $\alpha=0.91$ and second round's $\alpha=0.94)$; while 'using technology' had the lowest score (first round's $\alpha=0.65$ and second round's $\alpha=0.69$ ).

The competencies emphasized the most were in nutrition science (nine out of fourteen competencies); and the least were in the leadership area (one out of nine competencies). There was no association between the respondents' job position and their responses. In the first round, more than $25 \%$ of the panellists considered sixteen out of sixty-five items as essential and in the second round, $27 \%$ considered twenty-one out of seventy-seven competencies as essential.

The smallest difference (round 1 (58.5) - round 2 $(73 \cdot 8)=-15 \cdot 3)$ between the two rounds was seen in competencies related to the assessment and analysis area for the ability to provide nutritional and relevant information using the available data. On the other hand, the largest difference (round $1(52 \cdot 4)$ - round $2(29 \cdot 3)=+23 \cdot 1$ ) was seen in competencies related to the area of health and nutrition services used to control and regulate food advertisements.

Table 2 shows consensus development above $80 \%$ for the respondents answering in the first and second rounds on the essential competencies of public health nutritionists.

\section{Discussion and conclusion}

The present study investigated the opinions of Iranian experts regarding essential competencies required for effective PHN practice within the health-care system of Iran.
The highest consensus was on competencies related to nutrition science, the use of technology, and advocacy and community, while the lowest agreement was in areas related to planning and implementation of nutritional interventions, evaluation, and leadership and management.

Nutrition science is included as one of the main competencies of public health nutritionists worldwide ${ }^{(1,7)}$. However, according to Hughes et al.'s pyramid regarding the building blocks for effective $\mathrm{PHN}$ practice ${ }^{(8)}$, nutrition science is one of the required items in the level of critical competencies that is placed at a lower level. It is considered as a cognitive competency (knowing how). Moreover, the assessment and analysis and the evaluation domains were viewed as less necessary skills for public health nutritionists in our study. This is not consistent with the views expressed in other studies conducted in Australia and Canada ${ }^{(6,9)}$.

Searching for core competencies in PHN, eight formal competency sets were identified. Three were developed in Australia, which proposed seven to thirteen competency $\operatorname{areas}^{(6,10,11)}$, and others were developed in the $\mathrm{UK}^{(2)}$, Europe $^{(12)}$, the USA ${ }^{(13)}$ and Canada ${ }^{(9)}$. Despite the differences in definitions and terminology, major areas of knowledge and skills are almost similar. The agreement among PHN leaders in Europe, the USA and Australia regarding the effective competencies required for public health nutritionists has also been presented recently ${ }^{(6,12-15)}$.

The opinions of Iranian key experts regarding core competencies, general knowledge and skills required for public health nutritionists are also in concordance with those of their international counterparts (Table 3). Conversely, Iranian public health experts place less emphasis on planning, management and the analytical aspects that can be explained as follows. First, PHN is relatively a young area of nutrition in Iran; therefore, it is not well defined either academically or in the workplace. The initiation of Iran's present primary health-care system 
Table 2 Consensus development above $80 \%$ by respondents in the first and second rounds of the Delphi study on essential competencies of public health nutritionists; Iran, February-October 2012

\begin{tabular}{|c|c|c|c|c|c|c|}
\hline \multirow[b]{3}{*}{ Competency } & \multicolumn{4}{|c|}{$\geq 80 \%$ response rate } & \multirow{2}{*}{\multicolumn{2}{|c|}{$\begin{array}{c}\text { Shift } \\
\text { (round } 1-\text { round 2) }\end{array}$}} \\
\hline & \multicolumn{2}{|c|}{ First round } & \multicolumn{2}{|c|}{ Second round } & & \\
\hline & $n$ & $\%$ & $n$ & $\%$ & $n$ & $\%$ \\
\hline \multicolumn{7}{|l|}{ Nutrition science } \\
\hline Ability to recognize health-related issues and factors affecting the health and well-being of the population & 39 & $92 \cdot 9$ & 42 & $100 \cdot 0$ & 3 & $7 \cdot 1$ \\
\hline Knowledge of prevention, promotion and protection of population nutritional health & 41 & $97 \cdot 6$ & 41 & 97.6 & 0 & 0.0 \\
\hline Knowledge of health and nutrition indicators & 40 & $95 \cdot 2$ & 40 & $95 \cdot 2$ & 0 & 0.0 \\
\hline Knowledge of food composition and food sources of nutrients (macro- and micronutrients) & 39 & $92 \cdot 9$ & 39 & $92 \cdot 9$ & 0 & 0.0 \\
\hline Understanding the impact of lifestyle on protecting and promoting health and preventing diseases & 40 & $95 \cdot 2$ & 40 & $95 \cdot 2$ & 0 & 0.0 \\
\hline Knowledge of dietary requirements by age and sex and for nutritionally vulnerable groups & 41 & 97.6 & 40 & $95 \cdot 2$ & -1 & $-2 \cdot 4$ \\
\hline Knowledge of food and nutrition monitoring and surveillance systems & 34 & 81.0 & 35 & 83.3 & 1 & $2 \cdot 4$ \\
\hline Knowledge of necessary nutritional guidelines, health and nutrition policies, and related strategic plans at the national level & 35 & 83.3 & 35 & $83 \cdot 3$ & 0 & 0.0 \\
\hline Knowledge of common mistakes on nutrition issues in the community & 37 & 88.1 & 36 & 85.7 & -1 & $-2 \cdot 4$ \\
\hline Knowledge of the principles of nutrition & - & - & 37 & $90 \cdot 2$ & - & - \\
\hline Knowledge of assessing anthropometric parameters affecting public health & - & - & 37 & $90 \cdot 2$ & - & - \\
\hline \multicolumn{7}{|l|}{ Health and nutrition services } \\
\hline Education on healthy nutrition and using behavioural change techniques & 40 & $95 \cdot 2$ & 35 & $83 \cdot 3$ & -5 & -11.9 \\
\hline \multicolumn{7}{|l|}{ Advocacy and communication } \\
\hline Ability to achieve the cooperation of people in nutrition and health promotion programmes & 34 & $81 \cdot 0$ & 34 & $81 \cdot 0$ & 0 & 0.0 \\
\hline Ability of written communication skills & - & - & 34 & $81 \cdot 0$ & - & - \\
\hline \multicolumn{7}{|l|}{ Assessment and analysis } \\
\hline Ability to identify relevant and appropriate sources of information & 26 & 61.9 & 35 & 83.5 & 9 & 21.4 \\
\hline Ability to provide scientific and documented reports on an existing situation & 36 & $85 \cdot 7$ & 38 & 90.5 & 2 & 4.8 \\
\hline Identifying the target groups in different health and nutrition programmes & 36 & $85 \cdot 7$ & 37 & $88 \cdot 1$ & 1 & $2 \cdot 4$ \\
\hline \multicolumn{7}{|l|}{ Cultural, social and political aspects } \\
\hline Promote and preserve local food culture and good nutrition habits & 32 & $76 \cdot 2$ & 34 & $81 \cdot 0$ & 2 & 4.8 \\
\hline Solve nutritional problems based on the community's culture & 33 & 78.6 & 35 & $83 \cdot 3$ & 2 & 4.8 \\
\hline \multicolumn{7}{|l|}{ Using technology } \\
\hline $\begin{array}{l}\text { Ability to use appropriate technology for effective communication and education (such as letters, analysis, documentation, } \\
\text { PowerPoint) }\end{array}$ & 32 & $76 \cdot 2$ & 37 & $88 \cdot 1$ & 5 & 11.9 \\
\hline $\begin{array}{l}\text { Ability to use current and emergent technologies in providing health services for the population, } \\
\text { like nutrition consultation or designing educational materials (pamphlets, posters, clips, etc.) }\end{array}$ & 35 & $83 \cdot 3$ & 36 & $85 \cdot 7$ & 1 & $2 \cdot 4$ \\
\hline \multicolumn{7}{|l|}{ Leadership and management } \\
\hline Providing progress reports based on budget and time & 32 & $76 \cdot 2$ & 34 & $81 \cdot 0$ & 2 & $4 \cdot 8$ \\
\hline
\end{tabular}


Table 3 Comparisons of core competency areas as identified by Iranian experts and other countries' experts

\begin{tabular}{|c|c|c|c|c|c|c|}
\hline \multirow[b]{2}{*}{ Essential/Core competency } & \multicolumn{6}{|c|}{ Country of origin } \\
\hline & Iran & Australia $^{(5,6)}$ & Canada $^{(9,20)}$ & $\mathrm{NSA}^{(2)}$ & Europe $^{(12)}$ & $U_{S A}^{(13)}$ \\
\hline Food and nutrition science/Foundation and theoretical & $\mathfrak{S}^{*}$ & $\checkmark$ & $\checkmark$ & & & $\checkmark$ \\
\hline Research and evaluation & $\checkmark$ & $\checkmark$ & $\checkmark$ & $\checkmark$ & $\checkmark$ & $\checkmark$ \\
\hline Communication skills and cultural competencies & $\boldsymbol{S}^{*}$ & $\checkmark$ & $\checkmark$ & $\checkmark$ & & $\checkmark$ \\
\hline 4. Management and strategic leadership for health and well being & $\checkmark$ & $\checkmark$ & $\checkmark$ & $\checkmark$ & & $\checkmark$ \\
\hline Surveillance/Assessment of the population's health and nutrition & $\checkmark$ & $\checkmark$ & & $\checkmark$ & & \\
\hline Policy development and advocacy/Aspects of cultural, social and political & $\mathfrak{J}^{*}$ & $\checkmark$ & $\checkmark$ & $\checkmark$ & $\checkmark$ & \\
\hline Public health and nutrition systems/Food and nutrition systems & $\checkmark$ & $\checkmark$ & $\checkmark$ & $\checkmark$ & & \\
\hline Nutrition education/Building capacity in nutrition education and guidance & & $\checkmark$ & $\checkmark$ & & $\checkmark$ & \\
\hline $\begin{array}{l}\text { 9. Intervention management/Planning and implementation of nutritional } \\
\text { interventions }\end{array}$ & $\checkmark$ & $\checkmark$ & & $\checkmark$ & $\checkmark$ & \\
\hline $\begin{array}{l}\text { 10. Community dimensions of practice skills/Partnership and collaboration/ } \\
\text { Ensuring healthy and safe environments }\end{array}$ & $\checkmark$ & & $\checkmark$ & & & \\
\hline 11. Using technology & $\mathfrak{J}^{*}$ & & & & & \\
\hline
\end{tabular}

NSA, Nutrition Society of Australia.

*Items with the highest consensus in Iranian experts (above $80 \%$ ).

dates back to the $1980 \mathrm{~s}^{(16)}$; however, PHN programmes and the profession were introduced in the late 1990s (International Union of Nutritional Sciences, unpublished results).

Second, based on an international consultation report (International Union of Nutritional Sciences, unpublished results), current academic nutrition training in Iran is limited and focused too narrowly to adequately address community and PHN needs. Furthermore, existing curricular nutrition programmes in Iran do not produce skills and competencies required for programme planning and public health activities (International Union of Nutritional Sciences, unpublished results). In general, most academic curricular programmes in Iran have focused mainly on technical knowledge and skills; and despite the interdisciplinary and multisectoral nature of food and nutrition, the programmes lack important skills such as management and leadership. In a study by Davari et al. (2013) to determine leadership educational needs among Iranian food science and technology specialists, both qualitative and quantitative research techniques revealed limitations in leadership skills among the nutrition specialists. Effective team building, evidence-based decision making, and creativity and innovation were found as the most limiting leadership capabilities in the curriculum. Benchmarking with two similar programmes in South-East Asia and Europe also showed that two of these skills, i.e. effective team building and creativity, possess important places in the programme schedules ${ }^{(17)}$.

It is worth noting that the training background of the respondents might have had an influence on their answers. The majority of the respondents had a $\mathrm{PhD}$ in nutritional science with a variety of academic backgrounds. Only a few of them had specific training in PHN.

Finally, low involvement of public health nutritionists in programme planning in the country is likely a reason behind the views expressed by the Iranian experts. Otherwise, given the key experts' agreement on other planning domains, such as advocacy as a required competency for the public health nutritionist, higher agreement on assessment and analysis and evaluation domains in order to better advocate programmes is expected.

To recoup such deficits and upgrade leadership capacity among young, promising, allied food and nutrition graduates and PhD candidates, the Iranian Nutrition and Food Leadership Program (INFLP) was conceived in 2007. The programme aims to develop key aspects of transformational leadership and has put emphasis on leadership knowledge and skills, such as effective communication, team building, crisis resolution and motivation, through its interactive workshop sessions ${ }^{(18)}$. Given this specific attention to leadership skills among the competency areas for public health nutritionists, our study's findings highlight the importance of holding such nutrition leadership programmes for public health nutritionists, especially those working in the health-care system. Meanwhile, the leadership educational programmes should suitably be linked with the nutrition community's needs.

Currently, patterns of population age distribution, mortality, lifestyle and causes of death in the country are changing. These demographic and epidemiological transitions require urgent action and new ways of thinking that go beyond the common skills of public health nutritionists. To take proper preventive approaches to reduce the risks associated with a sedentary lifestyle and improper nutrition in countries experiencing transitions, innovative approaches and new public-based skills are needed. These needs are well reflected in the Vander Learning Pyramid on essential competencies for public health nutritionists, categorized in four rows and eighteen clusters $^{(8)}$. According to this model, knowledge about basic sciences, including biological, environmental, behavioural, social, economic and political sciences, comprises the basic level of requirement for public health nutritionists, while at the next level, public health nutritionists should be able to apply knowledge on nutrition science, analysis techniques, 
food and nutrition systems, and nutrition education within the public health system. In the third level, they are expected to show competency in areas such as management, leadership, communication and specialization. The highest level requires competencies in areas such as nutrition assessment, monitoring and surveillance, capacity building, intervention and management. Therefore, more complex and applied competencies are emphasized as the levels of basic requirements increase.

An earlier study on Iranian nutrition experts' viewpoints about the academic curriculum for the Nutrition Science programme have shown similar results, with more emphasis on diet therapy in hospitals, enhancing the population's nutritional knowledge and educating individuals about proper dietary habits ${ }^{(19)}$ and less emphasis on applied and skill-based competencies. After about two decades, it seems that the attitude of the experts remains stagnant and PHN is still neglected.

To our knowledge, the present study is the first effort in assessing the viewpoints of various Iranian public health experts regarding the competencies required for public health nutritionist at the national level. The Delphi process is time consuming and can prove challenging with regard to coordination; however, the researchers initiated several follow-up telephone calls to save time and avoid loss of interest and diminished cohesiveness over time. Communicating through email and telephone calls helped to preserve the anonymity of the participants and to avoid self-censorship. This self-censorship can arise as a result of social pressure that exists in face-to-face meetings and negative group influences such as dominating members and political lobbying.

However, similar to other Delphi studies, the study has some limitations. The quality of the responses was dependent on the panel participants. We tried to decrease this limitation by selectively inviting recognized experts to the study. For future studies, planning focus group discussions after compiling the Delphi results may improve data interpretation and final conclusions.

Results of the present study can serve as a needs assessment for improving current training programmes for public health nutritionists that can lead to changes in the academic curriculum as well as on-the-job training programmes. Therefore, future efforts should be focused on capacity building and curriculum renewal in the country.

\section{Acknowledgements}

Acknowledgements: The authors would like to acknowledge the kind efforts of the panellists and those who participated in our study (names are in alphabetical order): Abasalti Z., Abdollahi Z., Alizadeh N., Allameh S., Amani R., Aminian M., Aminpour A., Ardalan G., Barakati H., Bondarianzadeh D., Derakhshandeh F., Eslami M., Ebrahimi Mamaghan M.,
Esfarjani F., Esmailzadeh A., Ghavamsadri M., Ghiasvand R., Hajifaraji M., Jafari S., Jazayeri A., Kalantari N., Karandish M., Kavehi A., Khosravi A., Maani S., Mahboob S., Minaei M., Mohammadi Nasrabadi F., Mohammadian S., Najjarzadeh A., Nobakht Haghighi F., Nouri Saeedloo S., Sadeghian Sharif S., Sadrzadeh H., Safavi M., Salehi F., Salarkia N., Sahebdel M., Shaykholeslam R., Siassi F., Tabatabaei M., Taheri P., Torabi P., Vafa M. and Vakili M. Financial support: This research received no specific grant from any funding agency in the public, commercial or not-for-profit sectors. Conflict of interest: None. Authorship: Preparing the proposal and questionnaire and data analysis were performed by F.S.-G., E.S., N.O. and F.M. Interviewing and data collection were done by F.S.-G. The manuscript was prepared by F.S.-G., E.S. and N.O. and edited by F.K. Ethics of buman subject participation: The ethics committee of Shahid Beheshti University of Medical Sciences approved the study's protocol. The participants completed and signed the consent forms for the study and were ensured that their responses would be confidential.

\section{References}

1. Hughes R (2003) Definitions for public health nutrition: a developing consensus. Public Health Nutr 6, 615-620.

2. Nutrition Society of Australia (2007) Specialist Competencies in Nutrition Science: Public Health Nutrition. Adapted from UK Nutrition Society (September 2006). http://www.nsa.asn. au/useruploads/files/registration_documents/specialist_comp etencies_in_nutrition_science_public_health.pdf (accessed May 2014).

3. Ghassemi H, Harrison G \& Mohammad K (2002) An accelerated nutrition transition in Iran. Public Health Nutr $\mathbf{5}$, 149-155.

4. Asgari F, Aghajani H, Haghazali M et al. (2009) Noncommunicable diseases risk factors surveillance in Iran. Iran J Public Health 38, 119-122.

5. Australian Government Office for Learning and Teaching (2011) Consensus on core public health nutrition functions and competencies. Report from the Curriculum Renewal in Public Health Nutrition (CRIPHN) Project. Delphi Study. http:// www.olt.gov.au/system/files/resources/PP10_1769_Yeatman_ delphi study_2013.pdf (accessed May 2014).

6. Hughes R (2003) Competencies for effective public health nutrition practice: a developing consensus. Public Health Nutr 7, 683-691.

7. Hughes R, Shrimpton R, Recine E et al. (2012) Commentary: Empowering our profession. $J$ World Public Health Nutr Assoc 3, 33-54.

8. Hughes R, Shrimpton R, Recine E et al. (2011) A Competency Framework for Global Public Health Nutrition Workforce Development: A Background Paper. World Public Health Nutrition Association; available at www.wphna.org

9. Chenhall C (2006) Competencies for Public Health Nutrition Professionals: A Review of Literature. http://www.phredredsp.on.ca/Docs/Reports/Public_Health_Nutrition_Compe tencies_Final_Literature_Review_Sept06.pdf (accessed May 2014).

10. Queensland Health (2011) Health Practitioner Core Competency Descriptor. Nutritionist Discipline Descriptor. http://www.docstoc.com/docs/38958348/CoreCompetencies-for-Public-Health (accessed May 2014). 
11. Yeatman H, Hughes R, Begley A et al. (2013) Final Report: Curriculum Renewal in Public Health Nutrition. Wollongong, NSW: University of Wollongong.

12. Jonsdottir S, Thorsdottir I, Kugelberg S et al. (2012) Core functions for the public health nutrition workforce in Europe: a consensus study. Public Health Nutr 15, 1999-2004.

13. Mixon H, Dodds J \& Haughton B (2003) Guidelines for Community Nutrition Supervised Experiences, 2nd edition. Public Health/Community Nutrition Practice Group, American Dietetic Association and the Association of Graduate Programs in Public Health Nutrition, Inc. http://www.phcnpg.org/docs/ Resources/GuideCommunityNutrSuperExp.pdf (accessed May 2014).

14. Haughton B \& George A (2007) The Public Health Nutrition workforce and its future challenges: the US experience. Public Health Nutr 11, 782-791.

15. Jonsdottir S, Hughes R, Thorsdottir I et al. (2011) Consensus on the competencies required for public health nutrition workforce development in Europe - The JobNut project. Public Health Nutr 14, 1439-1449.
16. Tavassoli M (2008) Iranian health houses open the door to primary care. Bull World Health Organ 86, 585-586.

17. Davari A, Rashidi A, Zali MR et al. (2013) Leadership capabilities among Iranian food science and technology specialists: educational needs assessment. Iran J Nutr Sci Food Technol 8, 269-278.

18. World Health Organization (2012) Iranian Food and Nutrition Leadership Program - Nutrition education - Adult men and women. Global Database on the Implementation of Nutrition Action (GINA). https://extranet.who.int/nutrition/ gina/en/node/6060 (accessed May 2014).

19. Omidvar N, Zanganeh S, Haj Mohhamadi $F$ et al. (1996) Polls of university faculty and experts in the field about status nutrition education in Iran. Presented at the Fourth National Congress of Nutrition, Tehran, Iran, 5-7 November 1996.

20. Toronto Public Health, Division Management Team (2003) Nutrition Services Redesign Project. Final report. http:// www.torontopubliclibrary.ca/detail.jsp?Entt=RDM2946233 $\& R=2946233$ (accessed May 2014). 